\title{
Acquired Hemophilia in an Elderly Patient with Carcinoma of the Ampulla of Vater
}

\author{
Leonidas Mavroeidis ${ }^{a, b} \quad$ Amalia Vassou ${ }^{c}$ George Zarkavelis ${ }^{a, b}$ \\ Alexandra Papadaki ${ }^{a, b} \quad$ Ioanna Mouzaki ${ }^{a}$ Panagiotis Ntellas ${ }^{a}$ b \\ Stefania Gkoura a, b Ioanna Gazoulia, b George Pentheroudakisa, b \\ aDepartment of Medical Oncology, University of Ioannina, loannina, Greece; bSociety for the Study \\ of Clonal Heterogeneity of Neoplasia (EMEKEN), loannina, Greece; 'Department of Hematology, \\ University of Ioannina, loannina, Greece
}

\section{Keywords}

Acquired hemophilia - Carcinoma of the ampulla of Vater - Paraneoplastic acquired hemophilia

\begin{abstract}
Acquired hemophilia is a rare autoimmune bleeding disorder related to the production of autoantibodies that inhibit clotting factor VIII or IX. The underlying cause can be autoimmune disease, malignancy, pregnancy, or medications, but it is most commonly idiopathic. Here we present the case of an 81-year-old patient with locoregionally relapsed periampullary carcinoma who presented with soft tissue hematoma and an abnormally elevated activated partial thromboplastin time (aPTT) in the presence of a normal prothrombin time. A diagnosis of acquired hemophilia was established. The patient was managed with immunosuppressive prednisone and cyclophosphamide plus immunoglobulin G. He also received a cycle of chemotherapy with gemcitabine and oxaliplatin, because the underlying malignancy was the cause of the bleeding disorder. Care was complicated by neutropenia and nosocomial fever, but the patient eventually showed signs of clinical stability, while the aPTT decreased 2-fold. The patient was successfully discharged from the hospital and continued treatment in outpatient care.
\end{abstract}

\section{Introduction}

Acquired hemophilia is a rare autoimmune bleeding disorder. In contrast to congenital hemophilia, which is caused by a deficiency in either clotting factor VIII or clotting factor IX, acquired hemophilia develops due to production of autoantibodies, known as inhibitors, 

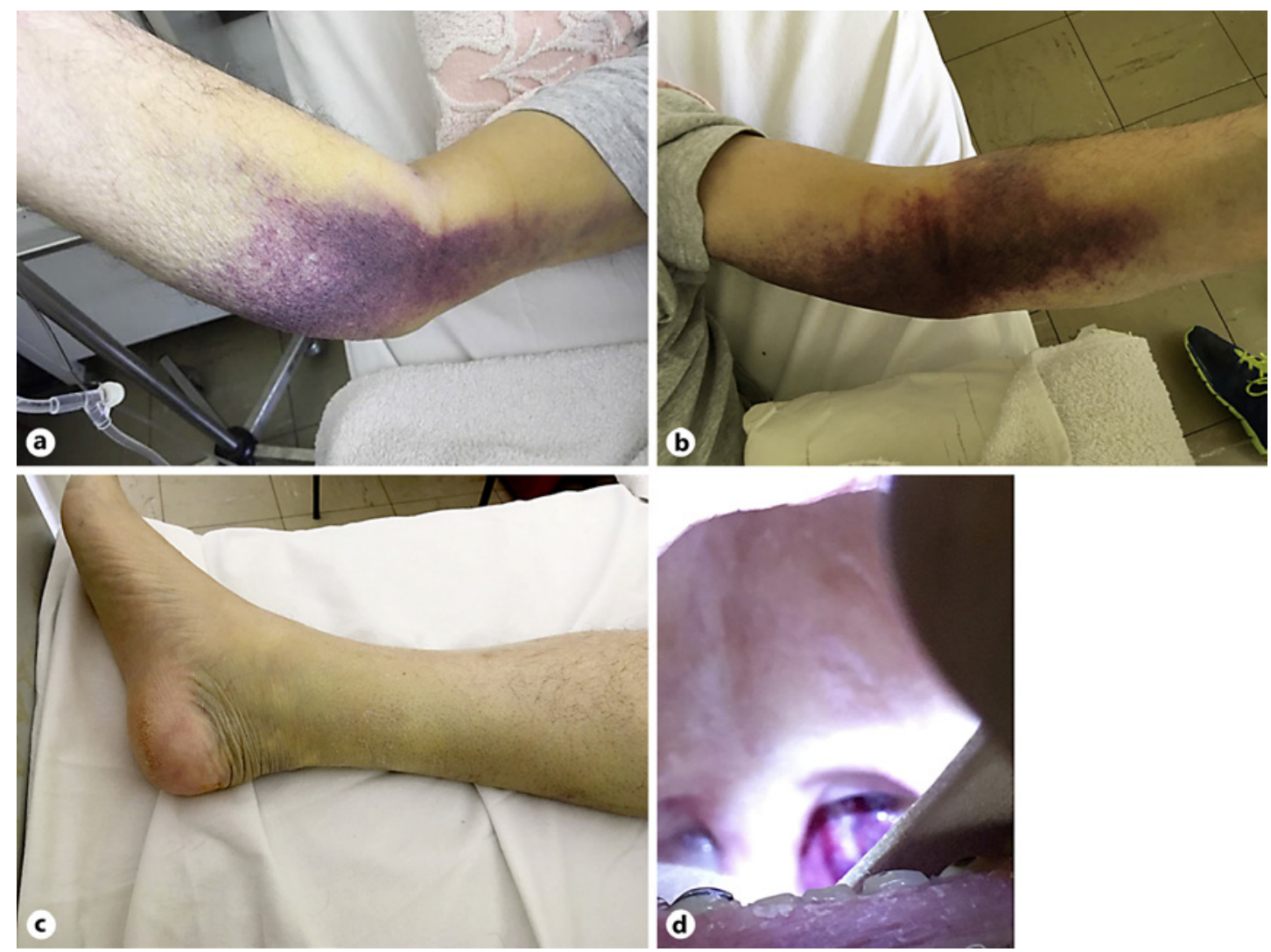

Fig. 1 Hematoma and ecchymosis. a Right forearm and arm. b Left forearm and arm. c Right shin. d Retropharyngeal hematoma.

most commonly against clotting factor VIII. Its incidence is 1-4 per million per year, and it usually presents in the elderly. Most cases are idiopathic (almost 50\%), but other conditions may be related as well, such as pregnancy, malignancy, autoimmune disease, and certain medications [1].

Acquired hemophilia usually manifests as soft tissue hematoma [2]. Bleeding in other sites may occur, such as in the gastrointestinal and genitourinary tract, while bleeding during invasive procedures may also take place. Unlike in congenital hemophilia, hemarthroses are rare. The laboratory hallmark is prolongation of the activated partial thromboplastin time (aPTT) in the presence of a normal prothrombin time. Other causes of aPTT prolongation should be excluded, such as antiphospholipid syndrome and heparin use [3]. Screening for inhibitors is the next step in establishing the diagnosis. aPTT is measured after mixing the patient's plasma with pooled normal plasma. Correction of aPTT indicates a factor deficiency or von Willebrand disease, whereas consistent prolongation suggests the presence of an inhibitor. In case the aPTT is corrected with the addition of phospholipids, a diagnosis of antiphospholipid syndrome is established. If the aPTT is not corrected, the next step is the Bethesda assay to confirm and quantify the titer of the inhibitor of factor VIII.

Treatment of acquired hemophilia consists of two axes [4]. Firstly, management of bleeding by administering a clotting factor with bypassing activity, and secondly, clearance of the inhibitor by using immunomodulatory agents. The most common agents are prednisolone and cyclophosphamide alone or in combination, while rituximab and immunoglobulin are also options. 
Fig. 2 Activated partial thromboplastin time (aPTT) value during the course of the treatment. Treatment was initiated with prednisone, cyclophosphamide, and immunoglobulin G. Then, a cycle of gemcitabine/oxaliplatin chemotherapy was performed on day 14 , after which there was a temporary discontinuation of cyclophosphamide due to fever and neutropenia.

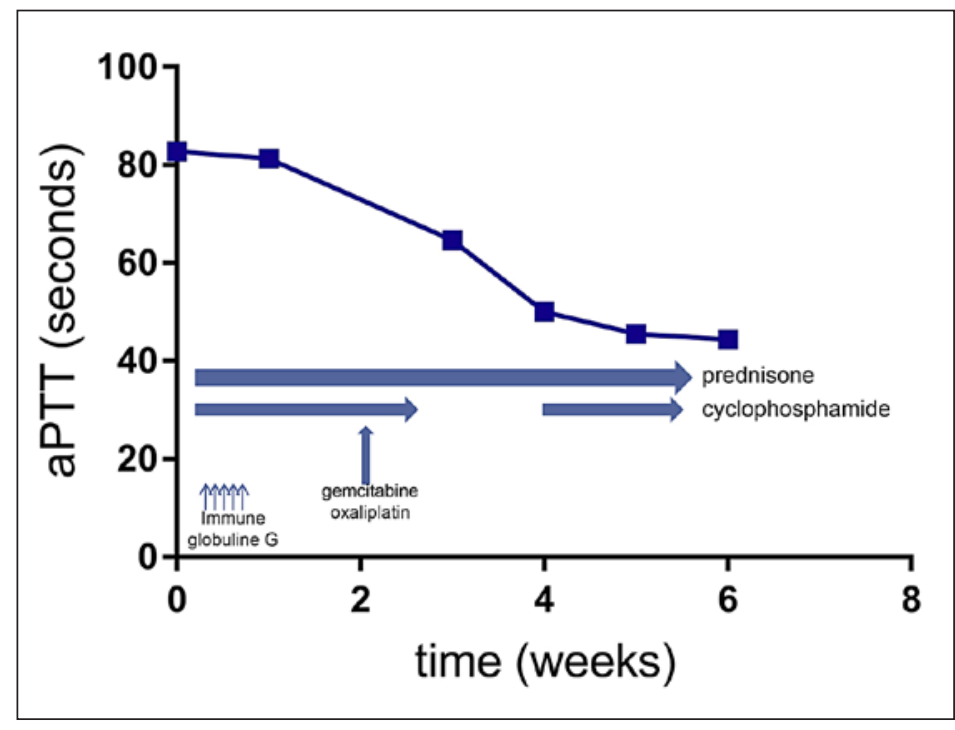

Here, we present the case of an elderly man with carcinoma of the ampulla of Vater who presented with soft tissue hematoma and a prolonged aPTT.

\section{Case Presentation}

An 81-year-old man presented due to presyncope to the emergency department. The past medical history included blood hypertension and Whipple's operation for carcinoma of the ampulla of Vater that had been staged as pT2N1M0 two months earlier. He had not received adjuvant chemotherapy but was on prophylactic low-molecular-weight heparin treatment.

The clinical examination revealed ecchymosis and edema of the right shin and the right and left forearms (Fig. 1). A digital rectal examination was negative for bleeding. The laboratory workup demonstrated normocytic normochromic anemia, slightly elevated lactate dehydrogenase, a prolonged aPTT with a normal prothrombin time, and fibrinogen with absence of schistocytes on a blood film. An ultrasound was performed due to enlargement of the right shin, which excluded thrombosis and confirmed the presence of hematoma. Differential diagnoses included an adverse reaction due to heparin use, antiphospholipid syndrome, and bleeding disorder due to the presence of an acquired inhibitor in the clotting cascade.

Heparin was discontinued and mixing studies were performed. Mixing with normal plasma did not correct the aPTT and the lupus anticoagulant test was negative. The presence of an acquired inhibitor of factor VIII was then suspected. Unfortunately, it was not possible to perform a Bethesda assay at the hematology laboratory of our hospital at that time due to lack of reagent. An investigation with immunological studies excluded other autoimmune diseases. A computed tomography scan of the abdomen was performed, exhibiting two peritoneal nodules compatible with metastasis. Therefore, his clinical condition was indirectly attributed to tumor recurrence.

Upon establishing a diagnosis of acquired hemophilia, immunosuppressive treatment was initiated (Fig. 2). The patient received prednisone IV (1 mg/kg per day), cyclophosphamide (100 mg p.o.), and immunoglobulin G IV ( $400 \mathrm{mg} / \mathrm{kg}$ for 5 days). Due to the association of this condition with carcinoma of the ampulla of Vater, the patient also received a cycle 
of chemotherapy with gemcitabine $\left(1,000 \mathrm{mg} / \mathrm{m}^{2} \mathrm{IV}\right)$ combined with oxaliplatin $\left(100 \mathrm{mg} / \mathrm{m}^{2}\right.$ IV) on day 14 of hospitalization. The patient continued treatment with prednisone $(1 \mathrm{mg} / \mathrm{kg}$ per day) combined with cyclophosphamide (100 mg per day p.o.) until signs of response were evident.

The patient had a prolonged hospitalization of 6 weeks. During his care, he complained of dysphagia, and laryngoscopy revealed a retropharyngeal hematoma (Fig. 1). This complication was managed with temporary discontinuation of alimentation and initiation of parenteral nutrition. Furthermore, care was complicated by nosocomial fever, which was effectively managed with piperacillin/tazobactam and vancomycin, while the patient later exhibited chemotherapy-induced neutropenia, managed with filgrastim. The aPTT decreased 2 -fold (Fig. 2), while the levels of factor VIII remained undetectable during the entire course of hospitalization. The patient showed signs of clinical stability and was successfully discharged for further outpatient care.

\section{Discussion and Conclusion}

Here, we presented a case of an elderly patient with acquired hemophilia, managed at the Department of Medical Oncology of the University of Ioannina, Greece. The patient's condition was attributed to tumor relapse. To the best of our knowledge, this is the first report of paraneoplastic hemophilia related to carcinoma of the ampulla of Vater [5].

Acquired hemophilia is an extremely rare manifestation of cancer, and neoplasia is one of the less frequent causes. Several malignancies have been reported to be associated with this condition [5]. The most common causes are lung cancer, prostate cancer, and lymphoproliferative diseases, while cases of gastrointestinal tumor as well as breast and bladder cancer have also been reported. Establishing a diagnosis is challenging without clinical suspicion. Sometimes the clinical presentation of the disease may precede cancer detection. One of the clinical hallmarks that prompt investigation is soft tissue hematoma in the presence of an abnormal aPTT [6].

The pathogenesis of acquired hemophilia is related to the development of autoantibodies against clotting factor VIII, which is an essential component of the intrinsic system of coagulation. These antibodies are polyclonal antibodies that bind to the A2, A3, or C2 domains of factor VIII, thus inhibiting its action and interfering with the activation of factor X, the initial step of the common pathway of coagulation [7]. The end result is aPTT prolongation not corrected after mixing with normal plasma, due to neutralization of factor VIII from the inhibitors. Regarding autoantibody production, this may be linked with the immune response in the tumor microenvironment [1]. Tumor cells release damage signals that trigger inflammation and phagocytosis. An antigen presentation process then leads to activation of an adaptive immune response against tumor neoantigens with shared epitopes with the antigens of the host.

Management of acquired hemophilia is based on three principles [4]. The first priority is treatment of bleeding. The second objective is elimination of the inhibitor, while the final goal is treatment of the underlying cause. Treatment of life-threatening hemorrhage is managed by administration of bypassing agents such as recombinant factor VIIa and activated prothrombin complex concentrate. Human factor VIII replacement therapy is only effective in patients with low titers of inhibitor ( $<5 \mathrm{BU})$. Invasive procedures should be avoided; otherwise, replacement therapy should be given before and after the procedure.

Immunosuppression is the mainstay of inhibitor elimination [8]. Effective regimens include prednisolone $(1 \mathrm{mg} / \mathrm{kg})$ monotherapy, combination of prednisolone $(1 \mathrm{mg} / \mathrm{kg})$ with cyclophosphamide (1-2 mg/kg p.o.), and rituximab administration with or without corticosteroids. The retrospective UKHCDO trial showed no statistically significant difference 
between corticosteroid monotherapy and combinational treatment [9], while a prospective study suggested that all patients should initially receive corticosteroids for 3 weeks and cyclophosphamide should be added if steroid resistance is noted [10]. However, we opted to treat this patient with a combination of corticosteroid and cyclophosphamide based on the results of the EACH2 registry, which demonstrated higher remission rates and a shorter time to remission with this regimen [11]. Although there is no proven added clinical benefit from retrospective studies, we also administered intravenous immunoglobulin as an adjunct based on results from case reports [12].

In addition to immunosuppression, treatment efficacy in cancer patients with acquired hemophilia is related to eradication of the tumor. In a systematic review, it was demonstrated that the best results regarding inhibitor elimination were achieved in patients who received effective treatment either by surgery or by chemotherapy or radiotherapy. By contrast, if cancer treatment was not successful, eradication of the inhibitor was not attained [5]. Our patient received a cycle of combinational chemotherapy with gemcitabine and oxaliplatin. We did not manage to administer more cycles during his hospitalization due to nosocomial infection and neutropenia. The patient was discharged after 6 weeks of hospitalization and was maintained on prednisolone (1 mg/kg) and cyclophosphamide (100 mg p.o.) with the aim to continue treatment for the underlying malignancy in the outpatient clinic. Although the patient was clinically stable, he was frail and never showed up at his appointment.

\section{Statement of Ethics}

Written consent was obtained from the relatives of the deceased patient for publication of this case report.

\section{Disclosure Statement}

The authors have no conflicts of interest to declare.

\section{Funding Sources}

The authors did not receive any funding.

\section{Author Contributions}

L.M. drafted the manuscript; L.M., A.V., and G.P. revised the manuscript; all authors were substantially involved in the conception and design of the case report, as well as in the acquisition, analysis and interpretation of the data; all authors approved the final version.

\section{References}

1 Sakurai Y, Takeda T. Acquired hemophilia A: a frequently overlooked autoimmune hemorrhagic disorder. J Immunol Res. 2014;2014:320674.

2 Collins P, Baudo F, Huth-Kühne A, Ingerslev J, Kessler CM, Castellano ME, et al. Consensus recommendations for the diagnosis and treatment of acquired hemophilia A. BMC Res Notes. 2010;3:161.

3 Tiede A, Werwitzke S, Scharf RE. Laboratory diagnosis of acquired hemophilia A: limitations, consequences, and challenges. Semin Thromb Hemost. 2014;40(7):803-11. 
4 Kruse-Jarres R, Kempton CL, Baudo F, Collins PW, Knoebl P, Leissinger CA, et al. Acquired hemophilia A: updated review of evidence and treatment guidance. Am J Hematol. 2017;92(7):695-705.

5 Napolitano M, Siragusa S, Mancuso S, Kessler CM. Acquired haemophilia in cancer: a systematic and critical literature review. Haemophilia. 2018;24(1):43-56.

6 Shetty S, Bhave M, Ghosh K. Acquired hemophilia A: diagnosis, aetiology, clinical spectrum and treatment options. Autoimmun Rev. 2011;10(6):311-6.

7 Ma AD, Carrizosa D. Acquired factor VIII inhibitors: pathophysiology and treatment. Hematology Am Soc Hematol Educ Program. 2006:432-7.

8 Charlebois J, Rivard GE, St-Louis J. Management of acquired hemophilia A: review of current evidence. Transfus Apher Sci. 2018;57(6):717-20.

9 Collins PW, Hirsch S, Baglin TP, Dolan G, Hanley J, Makris M, et al. Acquired hemophilia A in the United Kingdom: a 2-year national surveillance study by the United Kingdom Haemophilia Centre Doctors' Organisation. Blood. 2007;109(5):1870-7.

10 Green D, Rademaker AW, Briët E. A prospective, randomized trial of prednisone and cyclophosphamide in the treatment of patients with factor VIII autoantibodies. Thromb Haemost. 1993;70(5):753-7.

11 Collins P, Baudo F, Knoebl P, Lévesque H, Nemes L, Pellegrini F, et al. Immunosuppression for acquired hemophilia A: results from the European Acquired Haemophilia Registry (EACH2). Blood. 2012;120(1):47-55.

12 Lafferty TE, Smith JB, Schuster SJ, DeHoratius RJ. Treatment of acquired factor VIII inhibitor using intravenous immunoglobulin in two patients with systemic lupus erythematosus. Arthritis Rheum. 1997;40(4):775-8. 